\title{
SCIENTIFIC REPORTS

\section{OPEN Initial interaction of citrate-coated iron oxide nanoparticles with the glycocalyx of THP-1 monocytes assessed by real-time magnetic particle spectroscopy and electron microscopy}

\begin{abstract}
Wolfram C. Poller ${ }^{1,5,6^{*}}$, Norbert Löwa ${ }^{2,6^{*}}$, Moritz Schleicher ${ }^{1,5}$, Agnieszka MünsterWandowski ${ }^{3}$, Matthias Taupitz ${ }^{4}$, Verena Stangl ${ }^{1,5}$, Antje Ludwig ${ }^{1,4,5,6}$ \& Frank Wiekhorst ${ }^{2,6}$

Interaction with biological material can alter physicochemical parameters of magnetic nanoparticles and might thereby change their magnetic behavior with potentially important implications for various nanoparticle applications. Little is known about changes of the magnetic behavior that occur during the initial phase of cell binding and uptake. We investigate the magnetic behavior of very small superparamagnetic iron-oxide nanoparticles (VSOP) during initial contact with THP-1 monocytes. We combine real-time magnetic particle spectroscopy (MPS), a fast and sensitive method for specific detection of magnetic nanoparticles in biological specimen with high-pressure-freezing/freezesubstitution transmission electron microscopy (HPF/FS-TEM), enabling us to generate snapshots of the interaction of VSOP with the cellular glycocalyx. MPS reveals significant changes of the dynamic magnetic behavior within seconds afterVSOP injection into monocyte suspensions that correlate with the formation of nanoparticle clusters in the glycocalyx. The combination of real-time MPS and HPF/FSTEM provides an ideal platform to analyze magnetic behaviors of nanoparticles upon interaction with cells and tissues.
\end{abstract}

Magnetic iron oxide nanoparticles (MNP) serve in a broad spectrum of biomedical applications ranging from hyperthermia treatment of cancer over targeted drug delivery to contrast enhancement in magnetic resonance imaging (MRI) because of their special magnetic behavior in external magnetic fields ${ }^{1}$. This behavior is determined by the magnetic properties of MNP, which mostly depend on their composition, structure and size of the core. Additionally, physicochemical parameters such as surface coating and $\zeta$ potential as well as the mobility and aggregation state may further affect the magnetic behavior of MNP. As the interaction with biological material can alter the physicochemical parameters of the MNP their magnetic behavior may change as well ${ }^{2-5}$. While intermediate and long-term changes of MNP magnetism during the biodegradation process of cell-internalized MNP

\footnotetext{
${ }^{1}$ Charité - Universitätsmedizin Berlin, corporate member of Freie Universität Berlin, Humboldt-Universität zu Berlin, and Berlin Institute of Health, Medizinische Klinik mit Schwerpunkt Kardiologie und Angiologie, Campus Mitte, Charitéplatz 1, 10117, Berlin, Germany. ${ }^{2}$ Physikalisch-Technische Bundesanstalt, Abbestr. 2-12, 10587, Berlin, Germany. ${ }^{3}$ Charité - Universitätsmedizin Berlin, corporate member of Freie Universität Berlin, HumboldtUniversität zu Berlin, and Berlin Institute of Health; Institut für Integrative Neuroanatomie, Charitéplatz 1, 10117, Berlin, Germany. ${ }^{4}$ Charité - Universitätsmedizin Berlin, corporate member of Freie Universität Berlin, HumboldtUniversität zu Berlin, and Berlin Institute of Health; Institut für Radiologie, Campus Mitte, Charitéplatz 1, 10117, Berlin, Germany. ${ }^{5}$ DZHK (German Centre for Cardiovascular Research), partner site Berlin, Berlin, Germany. ${ }^{6}$ These authors contributed equally: Wolfram C. Poller, Norbert Löwa, Antje Ludwig and Frank Wiekhorst. *email: wolfram. poller@charite.de; norbert.loewa@ptb.de
} 
are relatively well investigated ${ }^{6-9}$, little is known about the change of the magnetic properties of MNP during the initial phase of cell contact and uptake.

Monitoring of the magnetic behavior of MNP during initial cell contact requires sensitive techniques with a high temporal resolution and the possibility to analyze living cells. Magnetic particle spectroscopy (MPS) was proven to be a fast, convenient and highly sensitive method for the specific detection of MNP in biological specimen ${ }^{10,11}$. By means of MPS the dynamic magnetic behavior of MNP under influence of an oscillating external magnetic field can be measured. The Fourier spectrum (An) of the measured temporal response is MNP specific and allows for sensitive quantification of MNP iron content, without being hampered by non-particular iron ${ }^{11}$. The amplitude and shape of the MPS spectrum is determined by many factors of influence. Apart from the main influential variables such as size distribution and magnetic anisotropy of the particles ${ }^{12}$, binding state ${ }^{13}$, viscosity $^{14}$, and interparticle interactions ${ }^{15}$, also modulate the spectral signature of MNP in MPS. The slope of the MPS spectrum can be parameterized by the concentration independent ratio of the fifth and third harmonic $\left(A_{5} / A_{3}\right.$ ratio $)^{5}$. Changes of the dynamic magnetic behavior (expressed by a change in $A_{5} / A_{3}$ ratio) caused by aggregation, viscosity changes or biodegradation can be assessed in biological systems whereby the extent of signal change strongly depends on the initial MNP size ${ }^{16}$. Importantly, MPS enables analyses in living cells.

Using MPS we have previously observed changes of the dynamic magnetic behavior of citrate-coated very small superparamagnetic iron oxide nanoparticles (VSOP), which have entered the clinical development up to phase II trials as a contrast agent for MRI ${ }^{17}$, during incubation with THP-1 monocytes ${ }^{2}$. The human monocytic cell line THP-1 is widely used to study monocyte/macrophage biology in cell culture systems. Monocytes and macrophages are cells of the innate immune system that have been shown to incorporate VSOP in in vivo and in vitro experiments ${ }^{2,18,19}$. In this previous study, we analyzed washed cell-pellets with MPS and observed a significant increase in the $\mathrm{A}_{5} / \mathrm{A}_{3}$ ratio of VSOP three minutes after the start of cell incubation, followed by a steady decrease towards the baseline level over the next $24 \mathrm{~h}^{2}$.

Before an MNP can enter a cell, it has to pass the glycocalyx (GCX), a membrane anchored polymer layer consisting mainly of proteoglycans and hyaluronic acid. For a long time, this structure was neglected, but recently it has been recognized how decisive the GCX is for cellular bioprocesses (e.g. cell migration, proliferation, adhesion) and for the uptake of nanoparticles ${ }^{20-22}$.

In the current study, we therefore hypothesize that the interaction of VSOP with the GCX of THP-1 cells during their very initial contact causes physicochemical alterations of the nanoparticles that are responsible for rapid changes of their dynamic magnetic behavior, i.e. the increase of the $A_{5} / A_{3}$ ratio, after cell contact.

To test our hypothesis, we conducted real-time in situ MPS measurements during the first seconds and minutes of incubation of THP- 1 cells with VSOP, to monitor changes of the $A_{5} / A_{3}$ ratio during the early VSOP-cell interaction without influencing the interaction by additional sample preparation steps. To visualize the association of VSOP with the GCX of THP-1 cells we used the technique of high-pressure-freezing/freeze-substitution transmission electron microscopy (HPF/FS-TEM), which was shown to largely preserve the hydrated configuration of the endothelial GCX ${ }^{23,24}$.

\section{Methods}

Very small superparamagnetic iron oxide particles (VSOP). All chemicals and solvents were obtained from Sigma-Aldrich, Germany, unless otherwise stated. VSOP used in this study were synthesized as previously described in detail ${ }^{2}$. The mean hydrodynamic diameter of the nanoparticles (as measured by laser light scattering) was $8.7-11.0 \mathrm{~nm}$ with a polydispersity index of 0.085 . The crystallite size measured by TEM (largest diameter of 500 crystals evaluated) was $6.8 \pm 2 \mathrm{~nm}$. The final ferrous iron ion content of $0.9 \%$ (molar ratio total iron) indicates nearly complete oxidation to maghemite. Selected area electron diffraction revealed a pure magnetite/maghemite pattern as previously published (PMID: 29017510).

Cell culture and VSOP incubation. THP-1 cells (ATCC, Germany) were cultured in suspension in RPMI medium (1640; Invitrogen, Germany) supplemented with fetal calf serum (10\% FCS, Biochrom, Germany), $100 \mathrm{U} / \mathrm{mL}$ penicillin (Invitrogen), $100 \mu \mathrm{g} / \mathrm{mL}$ streptomycin (Invitrogen), as described previously ${ }^{2}$.

In a first set of experiments, $10^{6} \mathrm{THP}-1$ cells suspended in $100 \mu \mathrm{L}$ of pre-warmed PBS were transferred to a glass tube and placed into the MPS spectrometer at $37^{\circ} \mathrm{C}$. After starting the MPS measurements, $100 \mu \mathrm{L}$ of a VSOP solution in PBS were added through a syringe and mixed briefly by repeated pumping, while the glass tube remained in the spectrometer and was continuously measured by MPS (Bruker, Germany). The experiments were conducted in quintuplicate using a VSOP iron concentration of $0.5 \mathrm{mmol} / \mathrm{L}$ and $2.4 \mathrm{mmol} / \mathrm{L}$, respectively. As control $100 \mu \mathrm{L}$ VSOP with $0.6 \mathrm{mmol} / \mathrm{L}$ iron were added to $100 \mu \mathrm{L}$ PBS without cells.

In a second set of experiments THP-1 cells were incubated with VSOP outside the spectrometer (ex situ), as described previously ${ }^{2,18}$. THP- 1 cells $\left(10 \mathrm{~mL}\right.$ with $5 \cdot 10^{6}$ cells $\left./ \mathrm{mL}\right)$ were incubated with VSOP $(0.5 \mathrm{mmol} / \mathrm{L}$ and $2.4 \mathrm{mmol} / \mathrm{L}$ ). After incubation for $300 \mathrm{~s}, 600 \mathrm{~s}$, and $900 \mathrm{~s}$ aliquots of $1 \mathrm{ml}$ each were collected and centrifuged for $1 \mathrm{~min}$ at $500 \mathrm{~g}$, and washed 2 times with PBS. Details of the analysis conditions are described below.

Magnetic particle spectroscopy. Magnetic particle spectroscopy (MPS) measurements on VSOP samples were performed using a commercial magnetic particle spectrometer (MPS-3, Bruker, Germany) as previously described in detail ${ }^{11}$. Primarily, this device is dedicated to assess the performance of MNP as tracers for a novel imaging modality called magnetic particle imaging (MPI). MPS specifically detects the non-linear magnetic response of MNP exposed to an oscillating magnetic field. Therefore, biological tissue and paramagnetic blood iron do not contribute to the MPS signal.

MPS measurements in standard operation mode were performed as described elsewhere ${ }^{6}$. To investigate the MNP interaction with cells in situ real-time MPS measurements were performed. Therefore, $10^{6}$ cells diluted in $100 \mu \mathrm{L}$ PBS were initially assembled in a glass tube (Bruker NMS PC 7.5). After placing the glass tube into the 
MPS pick-up coil repetitive measurements were started without VSOP to check for magnetic impurities. Over a time course of $10 \mathrm{~min}$ the MPS spectra were recorded every $4 \mathrm{~s}$ to improve the signal-to-noise-ratio. After the first measurements, $100 \mu \mathrm{L}$ of VSOP with an iron concentration of $c(\mathrm{Fe})=0.5 \mathrm{mmol} / \mathrm{L}$ and $2.4 \mathrm{mmol} / \mathrm{L}$ were added stabilized in PBS and mixed thoroughly (5 times). To transfer the sample from the pipette to the bottom part of the glass tube a flexible tubing (Rotilabo-FEP, Carl Roth $\mathrm{GmbH}$, Germany) was used to extend the tip. As a control the same procedure was applied to measure the time dependent behavior of $100 \mu \mathrm{L}$ VSOP $(c(\mathrm{Fe})=0.6 \mathrm{mmol} / \mathrm{L})$ after injection in medium (100 $\mu$ L PBS) only without cells.

High-pressure-freezing/freeze-substitution transmission electron microscopy. THP-1 cells were incubated with $1 \mathrm{mmol} / \mathrm{l}$ VSOP in RPMI medium supplemented with $10 \%$ fetal calf serum (FCS) for the indicated times ( $3 \mathrm{~min}, 30 \mathrm{~min}, 180 \mathrm{~min}$ ) followed by centrifugation of $1 \mathrm{ml}$ containing $10^{6} \mathrm{THP}-1 \mathrm{cells}$ ( $3000 \mathrm{rpm}$ for $2 \mathrm{~min}$ at room temperature). The cell pellet was re-suspended in $20 \mu \mathrm{l}$ RPMI medium, transferred into $3 \mathrm{~mm}$ copper gold-plated specimen carrier $(200 \mu \mathrm{m}$ indentation, Leica, Germany), coated with 1-hexadecene (Sigma-Aldrich, Germany) and immediately frozen in an EM ICE High Pressure Freezer (Leica, Germany) after reaching 2100 bar under liquid nitrogen. The detailed process of high-pressure freezing has been described previously ${ }^{23}$.

Freeze-substitution and embedding. Frozen samples were collected in liquid nitrogen and transferred into a freeze-substitution chamber (Leica EM AFS; Leica, Germany), where they were freeze substituted for $12-14$ hours at $-90^{\circ} \mathrm{C}$ in acetone containing $1 \%$ osmium tetroxide $\left(\mathrm{OsO}_{4}\right.$; Electron Microscopy Sciences, Germany) to enhance contrast and simultaneously stabilize the glycocalyx. Subsequently, the temperature was gradually increased $\left(5^{\circ} \mathrm{C} / 1 \mathrm{~h}\right)$ to $20^{\circ} \mathrm{C}$. Afterwards, the cells were centrifuged $(1000 \mathrm{rpm}, 3 \mathrm{~min})$, washed with acetone (Roche, Germany) and centrifuged again. After rinsing several times with acetone, the dehydrated samples were infiltrated first with HPMA (2-hydroxypropyl methacrylamide; Sigma-Aldrich, Germany), centrifuged at $3000 \mathrm{rpm}$ for $5 \mathrm{~min}$ and finally infiltrated overnight with HPMA-Epon mix (1:1). Suspension cells were further centrifuged at $5000 \mathrm{rpm}$ for $5 \mathrm{~min}$ and infiltrated with pure freshly prepared Epon for several hours. Finally, the samples were embedded in Epon and polymerized overnight at $60^{\circ} \mathrm{C}$.

Transmission electron microscopy. Ultrathin sections $(60 \mathrm{~nm})$ of embedded cells were cut using an ultramicrotome (Reichert Ultracut S, Leica, Germany) with a diamond knife (Diatome, Switzerland) and mounted on 300-mesh copper grids (Plano, Germany). Sections were examined, without further staining, using a Zeiss transmission electron microscope 912 (TEM-912, Carl Zeiss, Germany) operating at $80 \mathrm{kV}$ and equipped with a digital camera (Proscan $2 \mathrm{~K}$ Slow-Scan CCD-Camera, Carl Zeiss, Germany). Digital image acquisition was performed using the iTEM software (Olympus GmbH, Germany) ${ }^{2,18}$.

Data analyses. All MPS experiments were performed in quintuplicate, unless otherwise stated. Were appropriate, data are presented as mean \pm standard deviations with a coverage factor of $k=1$ (i.e. a confidence level of about 68\%). The limit of detection was determined according to guidance of the International Union of Pure and Applied Chemistry (IUPAC) as mean $+3 \mathrm{x}$ standard deviation of empty sample holder measurements with a coverage factor of $\mathrm{k}=1$ as well.

\section{Results}

Analysis of pre-conditions for a real-time in situ MPS monitoring. We first analyzed in which concentration range VSOP can be detected by MPS. Therefore, serial dilutions of VSOP in liquid suspension (free VSOP) were measured by MPS and the limit of detection (LOD) of the third $A_{3}$ and fifth $A_{5}$ harmonic amplitude of the MPS signal was assessed. The amplitudes $A_{3}$ and $A_{5}$ of the MPS signal showed a highly linear dependency on the corresponding iron mass above their LOD of less than $200 \mathrm{ng}(\mathrm{Fe})$ (Fig. 1). Furthermore, the shape of the MPS spectrum, represented by the ratio of the fifth $A_{5}$ to the third $A_{3}$ harmonic amplitude, remains constant within the examined concentration range at $6 \%$. This further indicates that the dynamic magnetic behavior is not affected by changing concentrations of VSOP, which is a pre-condition for a real-time in situ MPS monitoring of VSOP during incubation with THP-1 cells. To detect the binding of VSOP to THP-1 cells as early as possible by real-time in situ MPS the addition of too much free VSOP should be avoided. Thus, the upper limit of $m(\mathrm{Fe})$ was estimated to be $20 \mu \mathrm{g}(\mathrm{Fe})$, which is the maximum VSOP uptake capacity of $10^{6} \mathrm{THP}-1 \mathrm{cells}^{2}$.

Time resolved MPS measurements detect significant changes in the dynamic magnetic behavior of VSOP immediately after cell contact. Figure 2a shows the results of ex situ MPS measurement of THP- 1 cells after incubation with VSOP for $300 \mathrm{~s}, 600 \mathrm{~s}$, and $900 \mathrm{~s}$. The $A_{5} / A_{3}$ ratio of cell bound VSOP has increased to about $8 \%$ already after $300 \mathrm{~s}$, irrespective of the VSOP concentration used for incubation. As the $A_{5} / A_{3}$ ratio resembles the magnetic properties of VSOP, this observation indicates rapid physicochemical changes during cell contact, which was reported previously for VSOP of a different formulation ${ }^{2}$.

In order to investigate the very first contact of VSOP with THP-1 cells, we took advantage of the capability of MPS to perform in situ real-time measurements at a minimum temporal resolution of $20 \mu \mathrm{s}$. Figure $2 \mathrm{~b}$ displays the temporal evolution of the measured $A_{5} / A_{3}$ ratio after injection of VSOP into cell medium (PBS) only. The mean $A_{5} / A_{3}$ ratio of VSOP in PBS was $5.8 \% \pm 0.2 \%$, which is close to the $A_{5} / A_{3}$ ratio of free VSOP determined previously. This value remains nearly constant (coefficient of variation $2 \%$ ) during the entire 12.5 minutes of real-time MPS scans. This control experiment proofs that VSOP were still freely mobile in the cell medium and the dynamic magnetic behavior of VSOP was not altered by the MPS measurement procedure itself (field amplitude, injection). Subsequently, a tube containing the THP-1 monocytes was placed into the MPS device and VSOP were directly added to this tube during ongoing MPS measurements. Figure $2 \mathrm{c}$ shows the resulting time course of the $A_{5} / A_{3}$ ratio, which was characterized by a continuous increase of the $A_{5} / A_{3}$ ratio for both amounts of added VSOP $\left(m_{1}\right.$ and $\left.m_{2}\right)$. For the lower amount $m_{1}$ of VSOP (filled blue circles) the $A_{5} / A_{3}$ ratio had already 


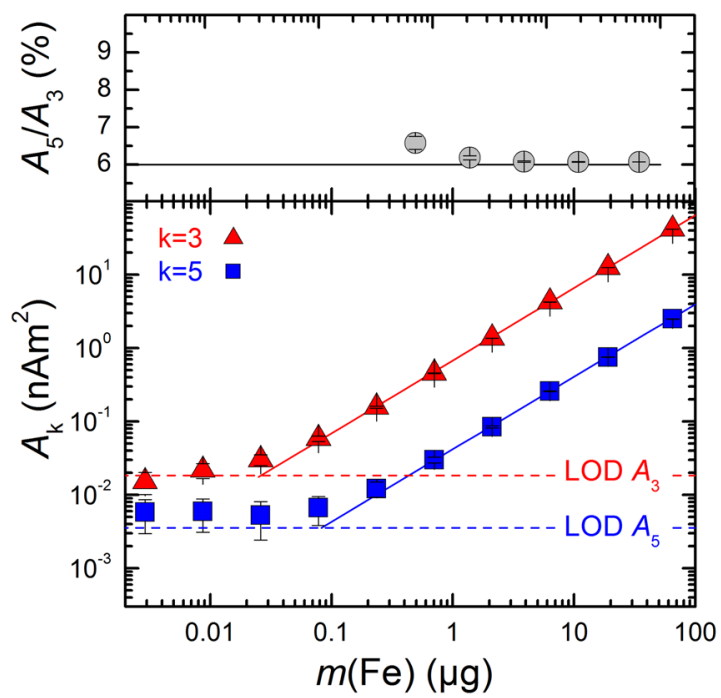

Figure 1. MPS measurements at $\hat{B}=25 \mathrm{mT}$ of VSOP in liquid suspension. MPS signals were acquired over a dilution range of the VSOP samples from $65 \mu \mathrm{g}(\mathrm{Fe})$ down to $3 \mathrm{ng}(\mathrm{Fe})$ iron. The uncertainty bars indicate the intrinsic noise of the MPS device determined by empty sample holder measurements $(n=100)$. The upper graph shows the ratio of the third A3 to the fifth A5 harmonic amplitude representing the concentration independent slope of the MPS spectrum (grey circles) showing a mean A5/A3 ratio of $6.05 \%$ (solid line). The lower graph displays that the signal amplitudes $A_{3}$ (red triangles) and $A_{5}$ (blue squares) are decreasing linearly with sample iron content. The limit of detection (LOD) of the respective harmonic amplitude results from 20 repeated measurements of an empty sample holder (dashed lines).

increased to $6.7 \% \pm 0.5 \%$ within the first $4 \mathrm{~s}$ after addition to THP- 1 cells. The mean $A_{5} / A_{3}$ ratio continuously increased throughout the entire incubation period and finally reached a value of $7.4 \% \pm 0.3 \%$ after 12.5 minutes, which corresponds to an increase of about $31 \%$ from baseline. The injection of a higher amount $m_{2}$ of VSOP resulted in a slightly lower change of the $A_{5} / A_{3}$ ratio (filled red circles), indicating a higher proportion of VSOP that remained unbound and freely mobile in the sample. With the higher amount $m_{2}$, the $A_{5} / A_{3}$ ratio finally reached a value of $6.83 \% \pm 0.07 \%$ (increase of $17 \%$ from baseline).

The quantified nanoparticular iron amount remained unchanged during the experiments as quantified by MPS and therefore a potential dissolution of the MNP can be excluded.

Changes of the magnetic behavior of VSOP immediately after cell contact correlate with VSOP compartmentalization and clustering in the glycocalyx. Imaging the interaction of the fragile glycocalyx with nanoparticles by TEM is challenging. Conventional sample preparation for TEM results in a nearly complete breakdown of the glycocalyx structure. By using HPF/FS-TEM we were able to visualize the glycocalyx of THP-1 cells as a several $\mu \mathrm{m}$ thick mesh-like structure (Fig. 3).

Figure 4 shows HPF/FS-TEM images of VSOP-labeled monocytes after the indicated incubation times at a high magnification to visualize the nanoparticles. At 3 minutes after the start of incubation, the majority of cell-bound VSOP were observed in small clusters distributed within the glycocalyx and as clusters directly on the cell membrane surface. Membrane invaginations associated with particle clusters indicated first endocytosis processes at this early time point of incubation. At later stages ( 30 minutes and 3 hours after incubation), the vast majority of cell-bound particles were found in cytoplasmic endosomes containing larger clusters of several hundred VSOP.

\section{Discussion}

In this study we observed a significant change of the dynamic magnetic behavior of VSOP during the initial contact of VSOP with THP-1 monocytes that correlated with compartmentalization and clustering of the nanoparticles in the glycocalyx.

This was achieved by taking advantage of the capability of MPS to perform in situ magnetic analyses of magnetic nanoparticles in a suspension with living cells and to monitor conformational changes in real time. Due to the different spectral signature of free (dispersed) and cell bound (clustered) VSOP, we were able to analyze the temporal change of VSOP states during incubation with THP-1 monocytes. Therefore, we used the $A_{5} / A_{3}$ ratio, which serves as a concentration independent parameter for the spectral signature of VSOP in MPS and allows to monitor the transition process from free to cell bound VSOP.

We observed a continuous increase of the MPS harmonic ratio starting from the value of dispersed (free) up to clustered (cell bound) particles when VSOP were injected into cell suspensions. Conversely, no MPS signal change was measured after injection of VSOP into cell medium (PBS) only.

Interestingly, we observed a change of the spectral signature of VSOP in MPS towards higher values of the $A_{5} / A_{3}$ ratio, which is contrary to the commonly observed decrease of the $A_{5} / A_{3}$ ratio that occurs during 
(a)

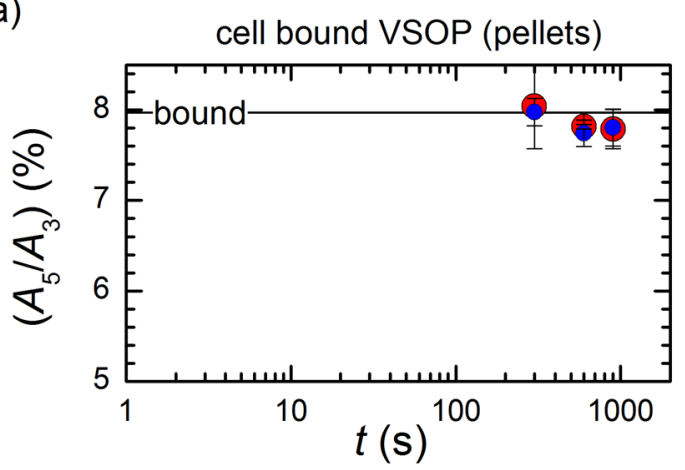

(b)

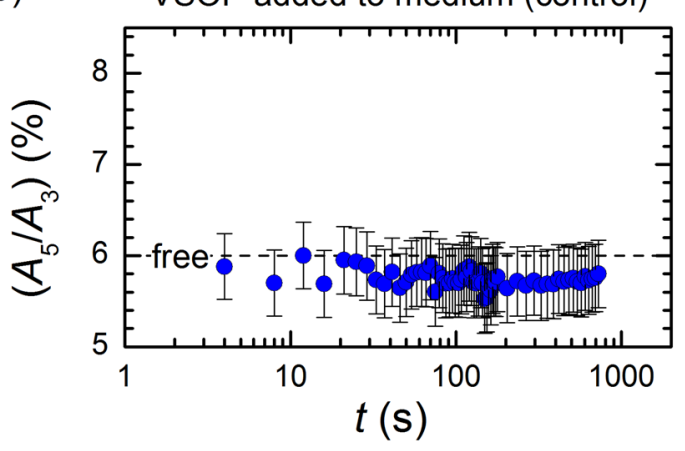

(c)

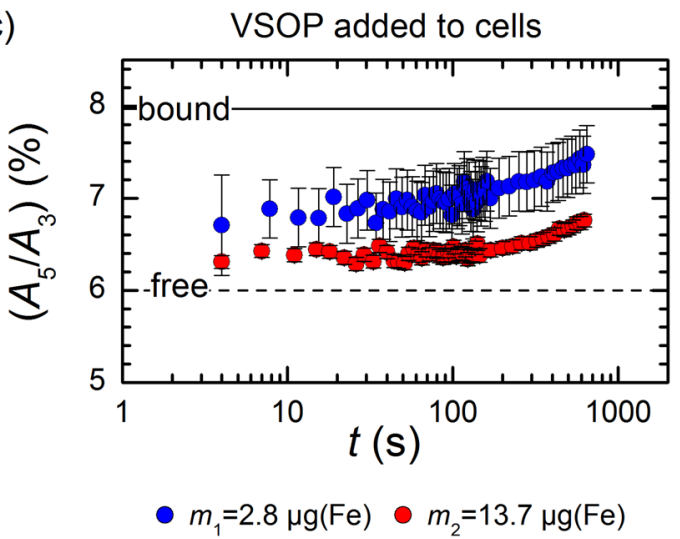

Figure 2. Cell binding and uptake of VSOP measured by MPS at $\hat{B}=25 \mathrm{mT}$. (a) The mean and standard deviation of the MPS signal of cell pellets taken after $300 \mathrm{~s}, 600 \mathrm{~s}$, and $900 \mathrm{~s}$ after start of VSOP incubation $(\mathrm{n}=3)$. The $A_{5} / A_{3}$ ratio significantly increases for cell bound VSOP regardless of the iron amount used for incubation (blue and red circles). (b) Time dependent MPS signal of VSOP directly injected into medium only (PBS). The $A_{5} / A_{3}$ ratio (blue circles) does not change with time compared to the initial state (short dashed line). The uncertainty bars indicate the standard deviation of five replicated experiments $(\mathrm{n}=5)$. (c) Time dependent MPS signal of VSOP directly injected into PBS with $10^{6}$ suspended THP-1 cells. A total iron amount of $2.8 \mu \mathrm{g}$ (blue circles) and $13.7 \mu \mathrm{g}$ (red circles) was used. A continuous increase of the $A_{5} / A_{3}$ ratio from the initial state to the bound VSOP state was observed depending on the applied iron amount. The uncertainty bars indicate the standard deviation of five replicated experiments $(\mathrm{n}=5)$.

progressive aggregation ${ }^{3,4,25}$ or cellular uptake ${ }^{26,27}$ of other MNP. This can be explained by the fact that such experiments were mostly performed with MNP of larger size and magnetic moment sufficient to deploy a rich harmonic spectrum in MPS, which is associated with a superior performance of these MNP in MPI. Magnetic dipolar interactions occurring between those large MNP in an aggregate lead to a reduced frequency response compared to non-interacting MNP, i.e. a lower spectral response and $A_{5} / A_{3}$ ratio in MPS. Due to the weak magnetic moment of the small sized VSOP, magnetic dipolar interactions have a positive effect on the frequency response. This was shown by Berkov et al. who found from numerical simulations that the magnetic dipolar interaction can either decrease or increase the frequency response of interacting MNP depending on the anisotropy of the individual MNP $\mathrm{MN}^{282}$.

Compared to our previously published experiments on washed pellets of VSOP-loaded cells ${ }^{2}$, we now detected significantly lower absolute values of the $A_{5} / A_{3}$ ratio both at baseline and after incubation with cells. As indicated 


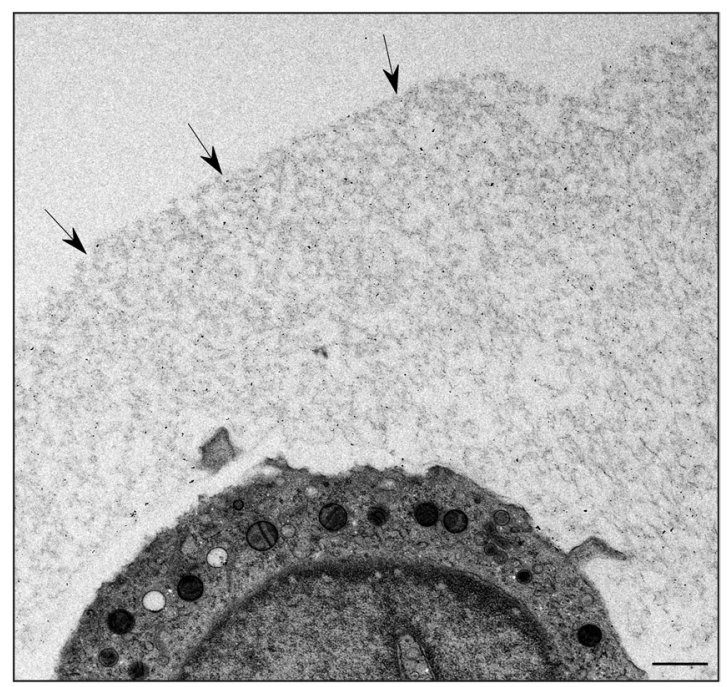

Figure 3. HPF/FS-TEM image showing the glycocalyx of a THP-1 monocyte after incubation with VSOP for 3 minutes. Black arrows indicate the outer border of the glycocalyx. Scale bar depicts $1 \mu \mathrm{m}$.

by their MPS spectral signatures, these differences in the absolute values between the VSOP batch used in the previous study ${ }^{2}$ and the currently used one might be caused by a different size distribution of the nanoparticles observed with DLS (PDI 0.39 and 0.107 for previous batch and new batch, respectively) with a higher content of larger entities in the previous batch. Importantly, however, the relative increase in the $A_{5} / A_{3}$ ratio between the unbound stage and the cell-bound stage is strikingly similar around 30\% for both VSOP batches $(18.1 \% \pm 0.1 \%$ to $24 \% \pm 1 \%$ and $5.8 \% \pm 0.2 \%$ to $7.4 \% \pm 0.3 \%$ for previous batch and new batch, respectively $)^{2}$, indicating that the same ultrastructural and magnetic alterations occurred.

Furthermore, the injection of a higher amount of VSOP resulted in a slightly lower change of the $A_{5} / A_{3}$ ratio. This indicates a higher proportion of VSOP that remained unbound and freely mobile in the sample. The observations from the ex situ experiments are in line with this hypothesis. For ex situ measurements, the cells were washed two times before the pellet was analyzed by MPS. These washing steps should have largely removed unbound nanoparticles, which have a lower $A_{5} / A_{3}$ ratio. Therefore, this approach leads to detection of the $A_{5} / A_{3}$ ratio of cell-bound VSOP only. Obviously, higher amounts of added VSOP do not seem to directly translate into higher amounts of VSOP in the glycocalyx. A potential explanation might be that VSOP only bind to specific domains within the glycocalyx or that changes in the electric charge distribution in the glycocalyx prevent further VSOP from entering the glycocalyx, so that only a certain amount of VSOP can be bound at a time. Another possibility is that, after a certain amount has been bound, it might just take more time for additional VSOP to enter the glycocalyx.

To correlate the observed MPS signal changes with morphological changes, we used the technique of high-pressure freezing with subsequent freeze substitution to fix our TEM samples. Chemical formaldehyde or glutaraldehyde based fixation methods inevitably induce strong ultrastructural changes that primarily effect water-rich labile components like the proteoglycans and glycosaminoglycans of the ECM, which is therefore almost completely lost during conventional fixation. HPF/FS-TEM overcomes these issues by simultaneously fixing all macromolecules in their hydrated biological configuration within milliseconds ${ }^{23}$. The rapid cooling process combined with the high pressure leads to the immediate formation of vitrified ice without any crystals ${ }^{23}$. In the subsequent freeze-substitution process, water is substituted by acetone and samples are slowly brought back to room temperature before the embedding process continues. Beside the much better ultrastructural preservation, HPF/FS-TEM also improves antigenicity for subsequent post-embedding immuno-labeling ${ }^{23}$. The major drawbacks of the method are its labor and cost expenses and the need for specific high pressure freezing and freeze substitution devices.

Using HPF/FS-TEM we were able to generate snapshots of the rapid and dynamic interaction of VSOP with the glycocalyx of THP-1 cells during the early cell contact ${ }^{24}$. For technical reasons the earliest time point that could be visualized was the state after 3 minutes. HPF/FS-TEM analyses revealed that the increase of the A5/A3 ratio temporally correlates with local clustering of VSOP in the glycocalyx of THP-1 cells in early phases, followed by the appearance of larger particle clusters in endosomes in later phases of VSOP incubation. As the MPS signal changed within seconds after injection of VSOP into cell suspension we suppose that VSOP clusters are formed in the glycocalyx immediately after initial contact.

The fast occurrence of VSOP clusters at the membrane and the prompt initiation of endocytosis of the particles, together with the results of real-time MPS point to a fast diffusion of a part of the VSOP through the glycocalyx without steric or electrostatic hindrance. Recent studies showed the size dependence of nanoparticle penetration into the glycocalyx and their accumulation there ${ }^{20}$. Lieleg et al. suggested a window of surface charges that enables a charged particle to diffuse within the glycocalyx without electrostatic hindrance ${ }^{30}$. With a hydrodynamic diameter of $\sim 10 \mathrm{~nm}$ and a zeta potential of $-25 \mathrm{mV}^{31}$ VSOP are obviously able to diffuse quickly into 


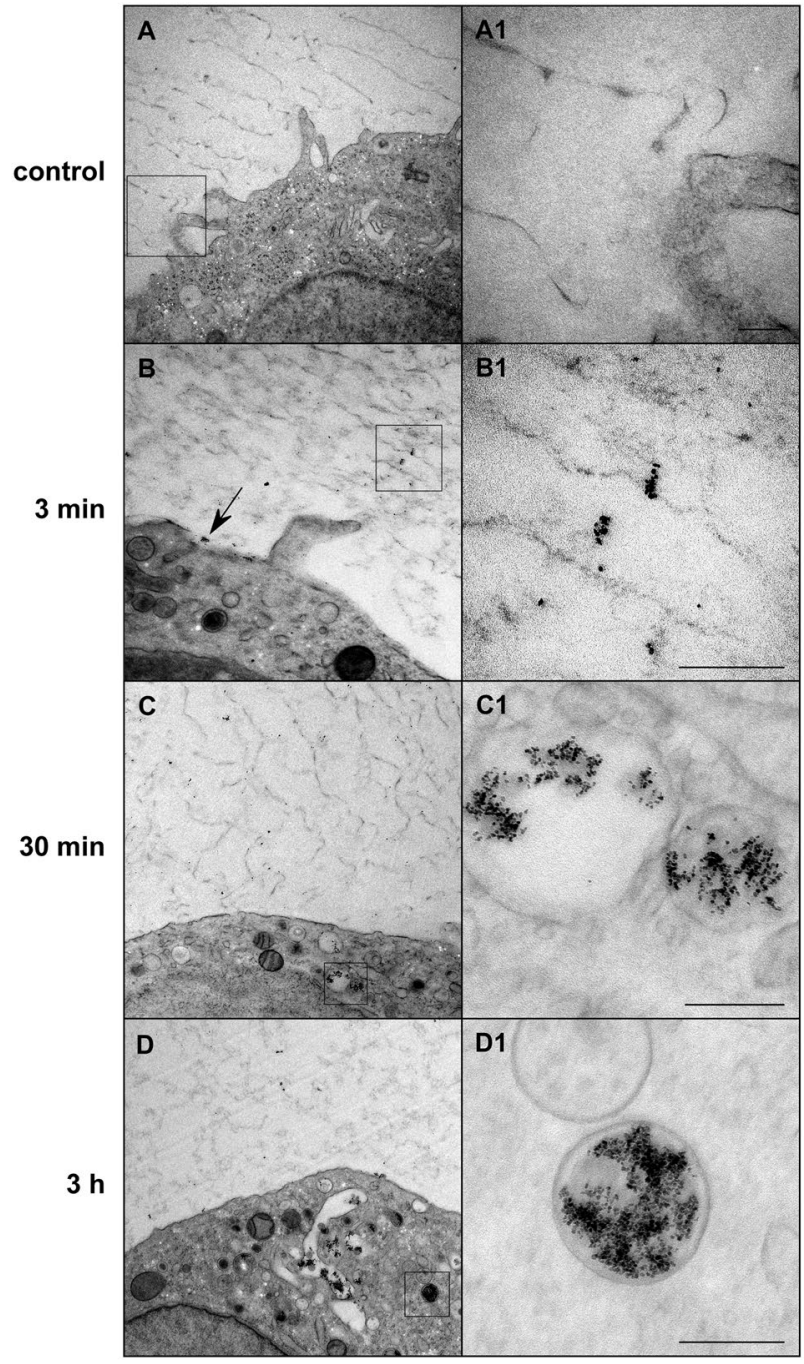

Figure 4. HPF/FS-TEM images showing the binding and uptake process of VSOP into THP-1 monocytes at the indicated incubation times. The control images A and A1 show a monocyte that was not incubated with VSOP. A1-D1 represent high-magnification images of the delineated area in A-D. VSOP are visible as black round structures with an individual nanoparticle diameter of $4-8 \mathrm{~nm}$. The black arrow in B points to a small VSOP aggregate bound to the cell surface membrane. Scale bars depict $200 \mathrm{~nm}$.

the glycocalyx. On the other hand, the clusters that were visible at certain sites of the glycocalyx could indicate that at least some of the VSOP interact with or bind to structures of the glycocalyx, such as proteoglycans and glycosaminoglycans (GAG). One possible interaction would be a transchelation reaction in which the citrate coat of the cationic iron oxide core of the VSOP is replaced by a GAG coat as hypothesized earlier ${ }^{2,18,32}$. GAG are strong chelators due to their high number of sulfation and carboxy groups and such a reaction was recently also proposed for citrate-coated gold nanoparticles, for which a high affinity to hyaluronic acid has been observed ${ }^{21}$. The glycocalyx of THP-1 cells has not yet been investigated in detail, however previous observations indicate that it mainly contains highly-chelating sulfated chondroitin sulfate $\mathrm{GAG}^{33}$.

To the best of our knowledge this study is the first description of a real-time MPS approach for live analyses of interactions between MNP and cells. The combination of real-time MPS and HPF/FS-TEM provides an ideal platform to analyze interactions of different MNP with the glycocalyx of various cell types.

Received: 21 August 2019; Accepted: 4 February 2020;

Published online: 27 February 2020

\section{References}

1. Krishnan, K. M. Fundamentals and Applications of Magnetic Materials. (Oxford University Press, 2016).

2. Poller, W. C. et al. Magnetic particle spectroscopy reveals dynamic changes in the magnetic behavior of very small superparamagnetic iron oxide nanoparticles during cellular uptake and enables determination of cell-labeling efficacy. J. Biomed. Nanotechnol. 12, 337-346 (2016).

3. Löwa, N., Seidel, M., Radon, P. \& Wiekhorst, F. Magnetic nanoparticles in different biological environments analyzed by magnetic particle spectroscopy. J Magn Magn Mater 427, 133-138 (2017). 
4. Arami, H., Ferguson, R. M., Khandhar, A. P. \& Krishnan, K. M. Size-dependent ferrohydrodynamic relaxometry of magnetic particle imaging tracers in different environments. Med Phys 40, 071904, https://doi.org/10.1118/1.4810962 (2013).

5. Rauwerdink, A. M. \& Weaver, J. B. Viscous effects on nanoparticle magnetization harmonics. J Magn Magn Mater 322, 609-613 (2010).

6. Löwa, N., Wiekhorst, F., Metzkow, S., Ludwig, A. \& Trahms, L. Magnetic Particle Spectroscopy for the Quantification of Magnetic Nanoparticles in Living Cells. Biomed Tech (Berl) 58 Suppl 1, https://doi.org/10.1515/bmt-2013-4138 (2013).

7. Mazuel, F. et al. Massive Intracellular Biodegradation of Iron Oxide Nanoparticles Evidenced Magnetically at Single-Endosome and Tissue Levels. ACS Nano 10, 7627-7638, https://doi.org/10.1021/acsnano.6b02876 (2016).

8. Mazuel, F. et al. In Tissue Engineering Part A Vol. 22 (ed. Inc Mary Ann Liebert) S107-S107 (2016).

9. Poller, W. C. et al. Very small superparamagnetic iron oxide nanoparticles: Long-term fate and metabolic processing in atherosclerotic mice. Nanomedicine 14, 2575-2586, https://doi.org/10.1016/j.nano.2018.07.013 (2018).

10. Gleich, B. \& Weizenecker, J. Tomographic imaging using the nonlinear response of magnetic particles. Nature 435, 1214-1217, https://doi.org/10.1038/nature03808 (2005).

11. Löwa, N. et al. In IEEE Trans Magn Vol. 49, no. 1 275,278 (2013).

12. Ludwig, F. et al. Characterization of magnetic nanoparticle systems with respect to their magnetic particle imaging performance. Biomed Tech (Berl) 58, 535-545, https://doi.org/10.1515/bmt-2013-0013 (2013)

13. Rauwerdink, A. \& Weaver, J. Measurement of molecular binding using the Brownian motion of magnetic nanoparticle probes. Appl. Phys. Lett. 96 (2010).

14. Draack, S. et al. Multiparametric Magnetic Particle Spectroscopy of CoFe2O4 Nanoparticles in Viscous Media. J. Phys. Chem. C 123, 6787-6801 (2019).

15. Wu, K., Su, D., Saha, R., Liu, J. \& Wang, J. Investigating the Effect of Magnetic Dipole-Dipole Interaction on Magnetic Particle Spectroscopy (MPS): Implications for Magnetic Nanoparticle-based Bioassays and Magnetic Particle Imaging (MPI). arXiv.org > physics $>$ arXiv 1901, 01355 (2019).

16. Teeman, E., Shasha, C., Evans, J. E. \& Krishnan, K. M. Intracellular dynamics of superparamagnetic iron oxide nanoparticles for magnetic particle imaging. Nanoscale 11, 7771-7780, https://doi.org/10.1039/c9nr01395d (2019).

17. Wagner, M. et al. Coronary MR angiography using citrate-coated very small superparamagnetic iron oxide particles as blood-pool contrast agent: initial experience in humans. J Magn Reson Imaging 34, 816-823, https://doi.org/10.1002/jmri.22683 (2011).

18. Ludwig, A. et al. Rapid binding of electrostatically stabilized iron oxide nanoparticles to THP-1 monocytic cells via interaction with glycosaminoglycans. Basic Res Cardiol 108, 328, https://doi.org/10.1007/s00395-013-0328-2 (2013).

19. Poller, W. C. et al. Uptake of citrate-coated iron oxide nanoparticles into atherosclerotic lesions in mice occurs via accelerated transcytosis through plaque endothelial cells. Nano Research 9, 3437-3452, https://doi.org/10.1007/s12274-016-1220-9 (2016).

20. Xu, R. et al. Pericellular matrix plays an active role in retention and cellular uptake of large-sized nanoparticles. Anal Bioanal Chem 406, 5031-5037, https://doi.org/10.1007/s00216-014-7877-6 (2014).

21. Zhang, S., Moustafa, Y. \& Huo, Q. Different interaction modes of biomolecules with citrate-capped gold nanoparticles. ACS Appl Mater Interfaces 6, 21184-21192, https://doi.org/10.1021/am506112u (2014).

22. Zhou, R., Zhou, H., Xiong, B., He, Y. \& Yeung, E. S. Pericellular matrix enhances retention and cellular uptake of nanoparticles. J Am Chem Soc 134, 13404-13409, https://doi.org/10.1021/ja304119w (2012).

23. Vanhecke, D., Graber, W. \& Studer, D. Close-to-native ultrastructural preservation by high pressure freezing. Methods Cell Biol 88, 151-164, https://doi.org/10.1016/S0091-679X(08)00409-3 (2008).

24. Ebong, E. E., Macaluso, F. P., Spray, D. C. \& Tarbell, J. M. Imaging the endothelial glycocalyx in vitro by rapid freezing/freeze substitution transmission electron microscopy. Arterioscler Thromb Vasc Biol 31, 1908-1915, https://doi.org/10.1161/ ATVBAHA.111.225268 (2011).

25. Rauwerdink, A. M. \& Weaver, J. B. Harmonic phase angle as a concentration-independent measure of nanoparticle dynamics. Med Phys 37, 2587-2592, https://doi.org/10.1118/1.3426294 (2010).

26. Arami, H. \& Krishnan, K. M. Intracellular performance of tailored nanoparticle tracers in magnetic particle imaging. J Appl Phys 115, 17B306, https://doi.org/10.1063/1.4867756 (2014).

27. Antonelli, A. et al. Red blood cells as carriers in magnetic particle imaging. Biomed Tech (Berl) 58, 517-525, https://doi.org/10.1515/ bmt-2012-0065 (2013).

28. Berkov, D. V. \& Gorn, N. L. Susceptibility of the disordered system of fine magnetic particles: a Langevin-dynamics study. J Phys Condens Matter 13, 9369 (2001).

29. Bervkov, D. V., Gorn, N. L. \& Görnert, P. Magnetization Dynamics in Nanoparticle Systems: Numerical Simulation Using Langevin Dynamics. Phys Status Solidi 189, 409-421 (2002).

30. Lieleg, O., Baumgartel, R. M. \& Bausch, A. R. Selective filtering of particles by the extracellular matrix: an electrostatic bandpass. Biophys J. 97, 1569-1577, https://doi.org/10.1016/j.bpj.2009.07.009 (2009).

31. Scharlach, C. et al. Synthesis of acid-stabilized iron oxide nanoparticles and comparison for targeting atherosclerotic plaques: Evaluation by MRI, quantitative MPS, and TEM alternative to ambiguous Prussian blue iron staining. Nanomedicine 11, 1085-1095, https://doi.org/10.1016/j.nano.2015.01.002 (2015).

32. Wagner, S. et al. Contrast-enhanced MR imaging of atherosclerosis using citrate-coated superparamagnetic iron oxide nanoparticles: calcifying microvesicles as imaging target for plaque characterization. International journal of nanomedicine 8, 767-779, https://doi. org/10.2147/IJN.S38702 (2013).

33. Ambrosius, M., Kleesiek, K. \& Gotting, C. Quantitative determination of the glycosaminoglycan Delta-disaccharide composition of serum, platelets and granulocytes by reversed-phase high-performance liquid chromatography. J Chromatogr A 1201, 54-60, https:// doi.org/10.1016/j.chroma.2008.06.007 (2008).

\section{Acknowledgements}

The project was funded by the Deutsche Forschungsgemeinschaft (DFG, German Research Foundation) - SFB 1340/1 2018, grant 372486779, projects A02 and B02, B03 and the Federal Ministry of Economics and Technology (BMWi) within the TransMeT project "Magnetische Messtechnik für die Größenfraktionierung magnetischer Nanopartikel". Additional funding was provided by the Friede Springer Herz Stiftung to WCP. We highly appreciate the excellent technical assistance of A. Stach and B. Söhl-Kielczynski. WCP was participant in the BIH-Charité Clinical Scientist Program funded by the Charité-Universitätsmedizin Berlin and the Berlin Institute of Health. We acknowledge support from the German Research Foundation (DFG) and the Open Access Publication Funds of Charité - Universitätsmedizin Berlin. 


\section{Author contributions}

W.C.P. and N.L. conceived the study, designed and conducted experiments, analyzed and interpreted data, prepared the figures and wrote the manuscript; M.S. performed experiments; A.M.W. provided materials; A.M.W., M.T. and V.S. provided intellectual input and worked on the manuscript, A.L. and F.W. conceived the study, designed and conducted experiments, analyzed and interpreted data, and wrote the manuscript. All authors have read and approved the final manuscript.

\section{Competing interests}

The authors declare no competing interests.

\section{Additional information}

Correspondence and requests for materials should be addressed to W.C.P. or N.L.

Reprints and permissions information is available at www.nature.com/reprints.

Publisher's note Springer Nature remains neutral with regard to jurisdictional claims in published maps and institutional affiliations.

(1) Open Access This article is licensed under a Creative Commons Attribution 4.0 International License, which permits use, sharing, adaptation, distribution and reproduction in any medium or format, as long as you give appropriate credit to the original author(s) and the source, provide a link to the Creative Commons license, and indicate if changes were made. The images or other third party material in this article are included in the article's Creative Commons license, unless indicated otherwise in a credit line to the material. If material is not included in the article's Creative Commons license and your intended use is not permitted by statutory regulation or exceeds the permitted use, you will need to obtain permission directly from the copyright holder. To view a copy of this license, visit http://creativecommons.org/licenses/by/4.0/.

(C) The Author(s) 2020 